\title{
FiveStar: Ultra-Realistic Space Experience System
}

\author{
Masahiro Urano ${ }^{1}$, Yasushi Ikei $^{1}$, Koichi Hirota ${ }^{2}$, and Tomohiro Amemiya ${ }^{3}$ \\ ${ }^{1}$ Tokyo Metropolitan University \\ 6-6, Asahigaoka, Hino-shi, Tokyo 191-0065, Japan \\ ikei@computer.org, urano@krmgiks5.tmit.ac.jp \\ ${ }^{2}$ Tokyo University \\ 5-1-5, Kashiwanoha, Kashiwa-shi, Chiba 277-8563, Japan \\ hirota@media.k.u-tokyo.ac.jp \\ ${ }^{3}$ Nippon Telegraph and Telephone Corporation \\ 3-1, Morinomiyawakamiya, Atugi-shi, Kanagawa 243-0198, Japan \\ amemiya.tomohiro@lab.ntt.co.jp
}

\begin{abstract}
This paper describes the development of the FiveStar system that provides five senses stimulations to the participant for the creation of ultrarealistic experiences. We performed an upgraded demonstration of the system to evaluate its individual technologies at Asiagraph 2010 in Tokyo. The content of the exhibit was the encounter with a yokai character that produces effects of extra-ordinary interaction between the participant and the imaginary characters. The experiences of participants were investigated as exploratory effort for the elucidation of this type of ultra-reality created in a fantasy world.
\end{abstract}

Keywords: Multiple-modality, Interactive Experience, Augmented Reality, Ultra Reality.

\section{Introduction}

Recently, a three-dimensional (3D) display has attracted people's attention since many manufacturers announced that a stereoscopic TV set will be distributed worldwide in the year of 2010. Several 3D movies have already been released from the movie makers that intend to shift the 3D technology from a theme park to theaters in a city and then to a living room. The 3D visual experience provided in such a movie is much more sophisticated as a whole than those created in the past. It gives more augmented sensation of presence than 2D movies with improved high-density digital image generation. However, the presence we get in the real environment is not created only with visual information but with all the modalities that make our lives truly real.

A new project on the development of a multimodal display has started in 2009 under the administration of Tokyo Metropolitan University. The project aims to develop a new display system that presents ultra realistic sensation by providing cues to multiple modalities [1]. The display system is named 'Five Senses Theater' (FiveStar) since it is intended to produce controlled stimuli to all human sensors, the five senses except for the gustatory sensation. The FiveStar is an interactive display system for a single user of home theater size. A three-dimensional visual display, a spatial sound, a haptic/tactile display, a wind and scent display, and a vestibular display are involved in the FiveStar that is targeted to produce a futuristic integrated 
ultra-reality [2] experience. To develop each component of the system properly, integration testbed contents were built first based on individual function arrangement with intuitive concept design.

\section{Five Senses Theater}

\subsection{Basic Configuration}

Figure 1 shows a design sketch of the Five Senses Theater submitted as a proposal at the beginning of the project. This illustrates hardware elements that form the system with their input data and output targeted sensations. Users get multiple-modality stimulations simultaneously from the system. The system consists of a stereoscopic visual display, a 7.1 channel surround audio system, a tactile and force feedback display system, a wind and scent delivery system, and a vestibular body motion system. These subsystems are integrated to perform an interaction with a participant along with a particular experience scenario the participant immerses in. The system is intended for a personal use hypermedia unit in the near future.

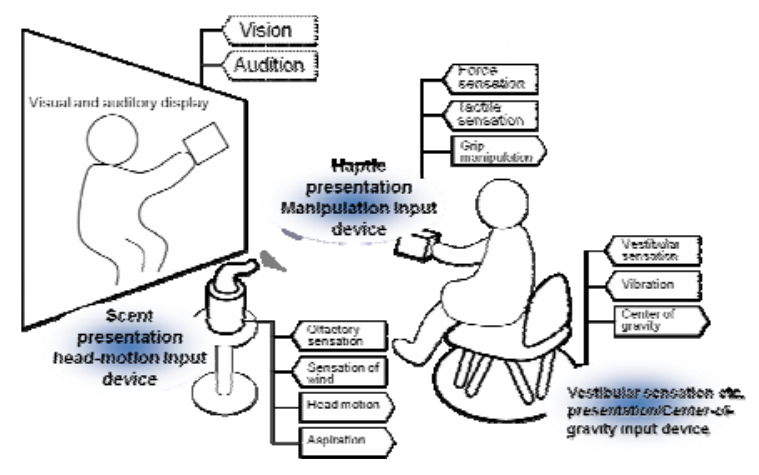

Fig. 1. First design sketch of Five Senses Theater. This is intended to present a particular kind of ultra reality. Multiple-modality sensations are integrated to render alternative reality and communication.

More specifically, the objective of the project is the development of core technology to build a platform for the five-sensory communication and five-sensory content experience. It aims to augment a uni-modal display device to an ultra-reality media with five-sensory presentation and interaction used in the communication. The target also includes the augmentation of rendering of experience of movies and video games.

For the creation of the ultra reality on the display device, we focus more on psychological effects than the fidelity of each device that has often technical limitation at present relative to the quality of human senses. Thus, what is to be pursued here is the effective rendering for impressive experience in which the impressiveness and richness of experience are the principal points of evaluation. Therefore, the basic idea is that the ultra-reality is not the extension of high fidelity-oriented reality. Of course the quality of presentation of each system is the bottom line. However, the integration of presentations in modalities to the user's intention is the point of the system. 


\subsection{Conceptual Level of Development}

To tackle with the ultra-reality, we assumed a multilevel structure for the FiveStar design. Figure 2 shows the conceptual levels that have three levels, Scene level, Sensation/Manipulation level and Device level. Actual interactions are exchanged at the Device level between sensors and motors of both sides of the user and the FiveStar. In this bottom layer, only limited information can be exchanged due to the limited capability of the physical device. The information at the level is described based on physical quantity.

The second level of Sensation and Manipulation level determines the range of device level to effectively produce the interaction performed by the user. The function of the second level is described by sensory information and motion input. The second level is designed to implement the upper level functions. The top level of Scene level handles the context to be exchanged through the FiveStar by the user. The scene is described at the cognitive and emotional points of views. We assume that the scene is rendered to best express or transfer the intention of the user. The five senses need to be integrated in this direction.

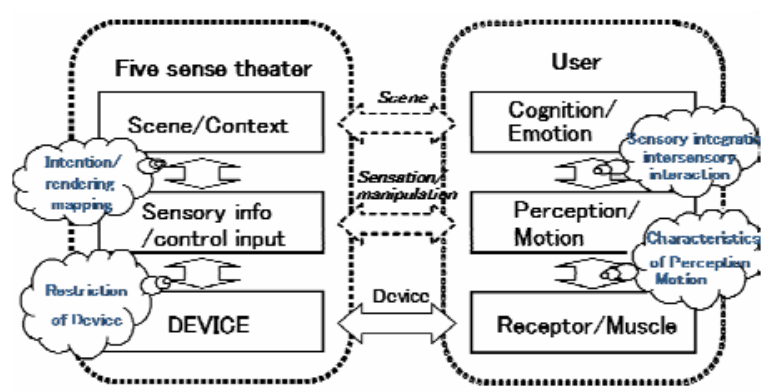

Fig. 2. Conceptual levels of Five Senses Theater design principle. Devices are only used to fulfill cognitive/emotional requirements placed essentially by the user.

\subsection{Challenges and Trials}

Challenges are how effectively transmit the impressive and eloquent information based on the multiple-modality interface devices. The presentation needs to be optimized on the cognition and interpretation level. For this purpose, we consider that stimulation is presented rather approximately than physically precisely. Also the input motion is interpreted rather approximately than investigated very precisely. The coherence of the objectives and the sensorimotor information exchange should be maintained in the interaction design. A support mechanism might be installed to control consistency.

For the embodiment of ultra-reality, we started investigation on the techniques to transmit reality more effectively than the usual approach that rebuilds the reality as it looks in the real world. The ultra-reality itself is an unestablished idea that will welcome an original proposal. We consider the ultra-reality has many aspects. Of course, it is more realistic than ever in a sense. But it is not necessarily higher definition reality than ever by higher resolution display. Probably, high resolution will not be solely sufficient for us. It is rather an issue of multiple-modality integration and rendition through the user's intention. 


\section{Prototype Exhibit at Asiagragh 2010}

The development of the each unit of every modality was focused so that the system could render a specific scene that worked as a bench mark of the whole system. The scenes were tested in technology exhibits where the prototype of FiveStar was first demonstrated in 2009 as an early stage evaluation of its element technology. Then, the prototype was improved and presented again as part of Asiagraph $2010^{1}$ in Tokyo. The original exhibit was named 'Ultra-ordinary experience through five senses media.' The content of the exhibit was an experience of a fantasy space: a participant who sat on the system was brown to an another dimension where a specter lived and casted evil magic particles to the participant. Then the system produced a multiplemodality interaction to the participant. This is one of approaches to embody and investigate how we think the ultra reality is. The reality has a number of aspects to be expressed differently by a media. The content of this exhibit was a trial to render the reality at the view of an imaginary world.

\subsection{System Configuration}

Figure 3 shows the configuration of the system. The system consists of a haptic (tactile and force) system to present physical reaction of a particular event, a stereoscopic visual system to render the world and to show the image of a participant using a stereo camera. A 7.1 surround audio, an eight-channel wind scent source system, a compressed air jet system, tactile stimulators to the body on the chair, a 3D motion seat, and a 2D feet motion generator system were also installed in the system to create integrated stimuli to the participant. Figure 4 shows the FiveStar system overview at the exhibit. The system was assembled at the site of the ARTEC exhibit in Asiagraph.

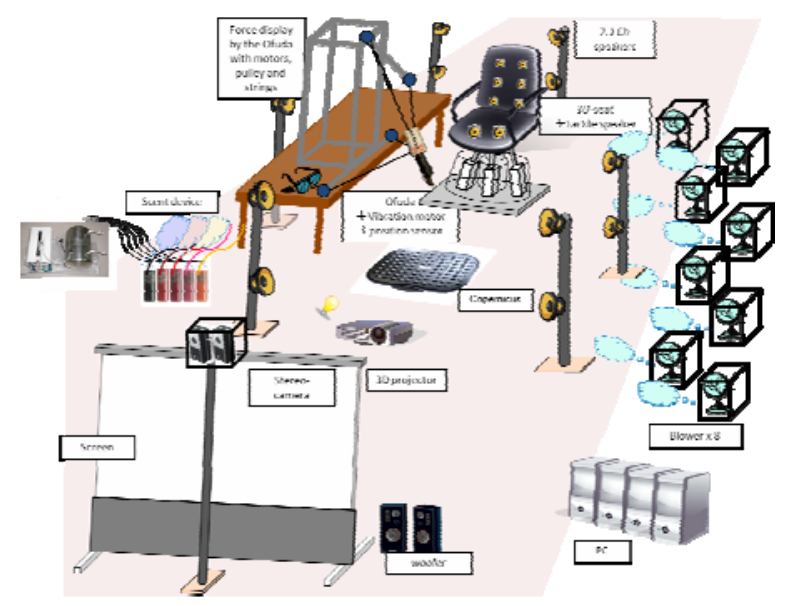

Fig. 3. System configuration of FiveStar exhibit in 2010

\footnotetext{
${ }^{1}$ National Museum of emerging science and innovation in Tokyo.
} 


\subsection{Theme of Exhibit}

The theme of demonstration we created was a short-time encounter experience with the 'Yokai' which is a Japanese traditional specter. The scenario of this experience consists of three parts. First, the participant was brown by a wind caused by a fanciful creature from the site to an another world. Then evil specter appeared casting the magic particles from its mouth. The participant protects the particles of magic by holding a shield with an amulet called 'ofuda'. After the participant received the magic particles sufficiently into the amulet, the participant could throw the amulet to the specter. When the amulet defeated the specter, it changed to a friendly character. Then the specter let the participant be back to the original world where the participant was sitting. Thus, a participant was able to experience and interact with an extraordinary world with multiple sensations.

\section{Displays of the System}

We built a personal interactive theater where the response from the simulated world was designed best to fit to the participant's imagination of the story. This is a working prototype that we assume a personal use hypermedia unit for the future. Figure 4 shows the system setup built at Asiagraph 2010.

\subsection{Haptic System for the Amulet (ofuda)}

The haptic system consists of a 3D force-feedback device which is called 'elePhantom' and a six-channel tactile device ('ofuda' device). The haptic system is shown in Figure 5. A participant held the ofuda device that presented reaction force by the elePhantom at the hand and tactile sensation on the palm during the interaction with the VR space.

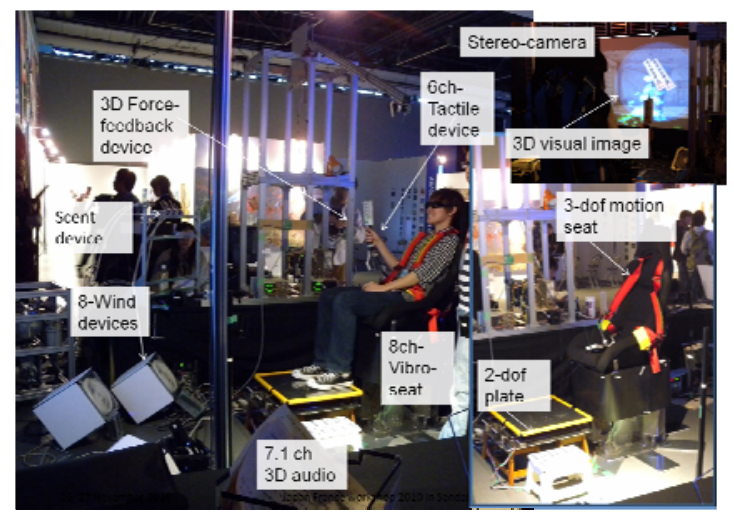

Fig. 4. System overview of FiveStar exhibit in 2010

The force feedback device (elePhantom) consists of four motors and strings connected to the ofuda device to suspend and pull it to add force vector to the hand of a participant. Figure 6 shows the ofuda device with tactile grip that installs six 
vibrators which provides tactile feedback sensation at the palm. The three dimensional position of the ofuda device is measured by the length of strings and its rotation by a spatial sensor. (Patriot, Polhemus)

In the course of the scenario of the VR space, the participant defended against the magic particles by a half-transparent shield with an amulet that is controlled by the ofuda device. The collision of particles caused force perturbation and impact and tactile stimulation on the device.

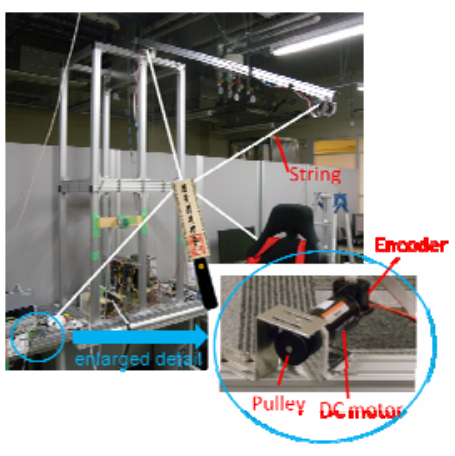

Fig. 5. Force feedback device

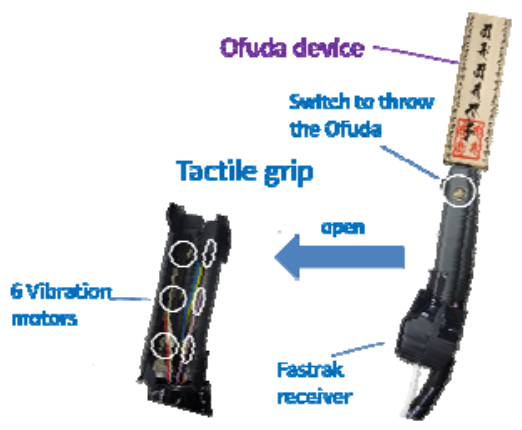

Fig. 6. Tactile feedback (ofuda) device

\subsection{D Motion Seat and 2D Feet Motion Generator System}

Somesthetic (bodily) sensation system consists of a 3-dof motion seat (Fig. 7, 3D Seat) and 2-dof feet motion system (Fig. 8, Copernicus). This system produces vestibular stimulation that changes the participant's attitude (head position) in addition to the body posture change by the feet motion relative to the seat. The system generates both the changes in head position/orientation and in those of feet.

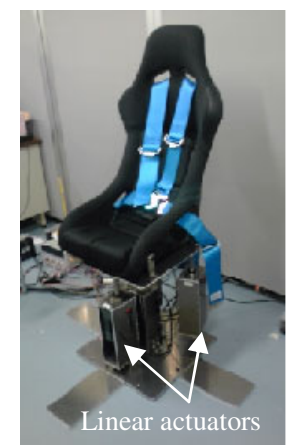

Fig. 7. 3D motion seat

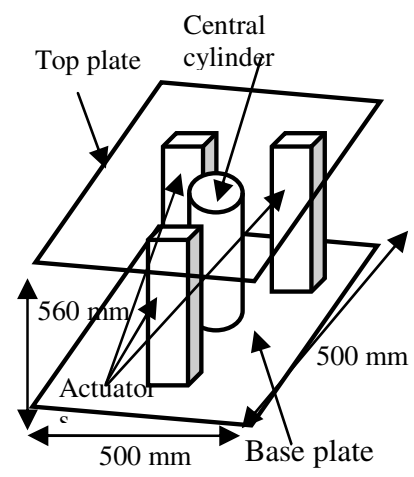

Fig. 8. Drive mechanism of the $3 \mathrm{D}$ motion seat

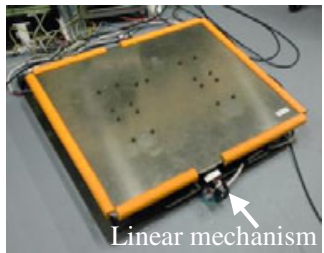

Fig. 9. 2D feet motion device (Copernicus) 
The 3D motion seat shown in Fig. 7 consists of three electric linear actuators and a central cylinder (Fig. 8). The stroke of the actuator is $100 \mathrm{~mm}$ and its max velocity is $200 \mathrm{~mm} / \mathrm{s}$. The possible motions are up-and-down, pitch, and roll motions. These motions are combined to generate multiple dof motions of the seat. The $2 \mathrm{D}$ feet motion device shown in Fig. 9 installs two motors that drive the top plate $100 \mathrm{~mm}$ to the $x-y$ directions in a horizontal plane where the participant puts the feet.

In the exhibition, the 3D seat and the feet device were driven simultaneously in accordance with the scenario to produce a whole body motion.

\subsection{Tactile Feedback of the Seat}

Tactile feedback was installed to work at the surface of the 3D motion seat (Fig. 10). The stimulator unit is a full-range speaker to create stimulations on the back and under the thigh of the seated participant to enhance the experience. Figure 10b shows two speakers installed in the bottom pad of the seat. Figure 10c shows six speakers in the back pad. These stimulators were driven so that the motion on the skin surface was properly created. In addition, the spatial sound of the particular scenario is mapped to enhance the effect of auditory information.

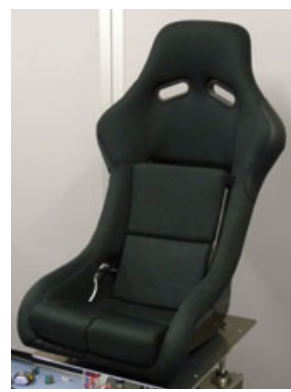

(a)

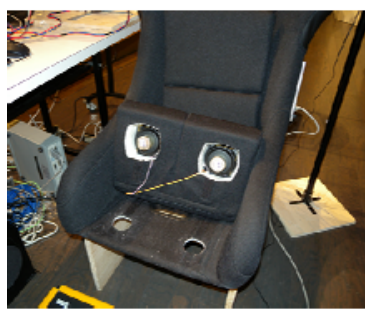

(b)

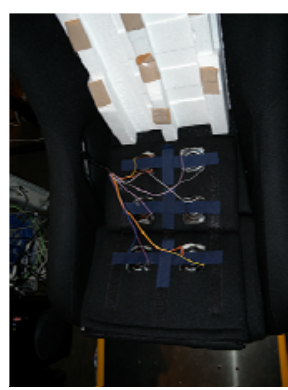

(c)

Fig. 10. a) Seat with tactile feedback, b) Stimulator units in the bottom pad, C) Six channel back vibrator unit

\subsection{Wind and Scent Display}

The stimulus of air flow to the face and upper body of a participant was controlled by eight wind fans (Figure 13). The fans were arranged in front of the participant with an appropriate interval to be able to change wind direction to his/her face. Eight to sixteen kinds of scent are able to be presented by the system. A scent of a scene is delivered by the compressed air that goes through one of the scent chambers which are filled with scent sources. The intensity of scent is determined flow volume by a valve control (Figure 15).

In the two exhibits, a scent display played a very important role since the questionnaire collected from the participants who had experienced with the demonstration rated that the scent was the most impressive among modalities. 


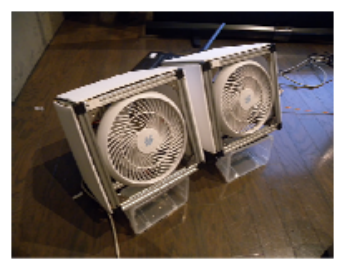

Fig. 11. Wind device

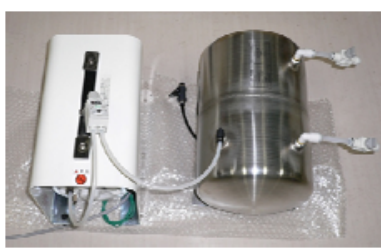

Fig. 12. Chamber and compressor

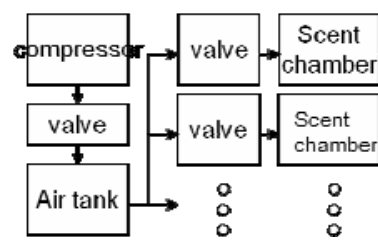

Fig. 13. System summary of the Scent display

\subsection{Sound Presentation}

A 7.1-ch sound was created around the participant with eight speakers. The sound of magic particles that the evil specter casted was presented at the position of particle obtained from VR environment software. In addition, some pieces of BGM music to the theme were composed and effectively enhanced the reality of the story.

\subsection{Visual Presentation}

The VR environment of this exhibition was rendered stereoscopically with a VR software, OmegaSpace (Solidray Co., Ltd.) and a stereo projector (Sight3D). The last scene which returns to the National Museum of Emerging Science was expressed by displaying realtime 3D image of the participant capturing with the stereo camera. The image was projected by a $3 \mathrm{D}$ projector and seen with shutter glasses.

\section{Conclusion}

The first stage implementation of Five Senses Theater (FiveStar) was investigated through the exhibition of Asiagraph. The rendered fantasy world was a good example to clarify how the multiple modality presentation was integrated effectively from the view point of ultrareality creation. We will continue to develop basic elements for multiple-modality presentation to improve the FiveStar performance. The interaction among modalities to enhance the whole effect of expression for communication and authoring content will be principal issue to pursue in this project hereafter.

Acknowledgments. This project has been supported by a grant for the ultra realistic communication technology by an innovative 3D image technique. We would like to thank National Institute of Information and Communications Technology, Japan.

\section{References}

1. Heilig, M.: Sensorama (1961), http: / / en.wikipedia .org/wiki/-Sensorama

2. Enami, K., Katsumoto, M., Nishimura, R.: Current techniques for Ultra-Reality and the future. The Journal of the Acoustical Society of Japan 64(5), 322-327 (2008) (in Japanese) 